\title{
Inhomogeneous free-electron distribution in InN nanowires: Photoluminescence excitation experiments
}

\author{
J. Segura-Ruiz, A. Molina-Sánchez, N. Garro, A. García-Cristóbal, and A. Cantarero \\ Institut de Ciència dels Materials, Universitat de València, E-46071 València, Spain \\ F. Iikawa \\ Instituto de Fisica Gleb Wataghin-Unicamp, CP 6165, CEP-13083970 Campinas, SP, Brazil \\ C. Denker, J. Malindretos, and A. Rizzi \\ IV. Physikalisches Institut, Georg-August Universität Göttingen, Göttingen, Germany
}

(Received 7 June 2010; published 17 September 2010)

\begin{abstract}
Photoluminescence excitation (PLE) spectra have been measured for a set of self-assembled InN nanowires (NWs) and a high-crystalline quality InN layer grown by molecular-beam epitaxy. The PLE experimental lineshapes have been reproduced by a self-consistent calculation of the absorption in a cylindrical InN NW. The differences in the PLE spectra can be accounted for the inhomogeneous electron distribution within the NWs caused by a bulk donor concentration $\left(N_{D}^{+}\right)$and a two-dimensional density of ionized surface states $\left(N_{s s}^{+}\right)$. For NW radii larger than $30 \mathrm{~nm}, N_{D}^{+}$and $N_{s s}^{+}$modify the absorption edge and the lineshape, respectively, and can be determined from the comparison with the experimental data.
\end{abstract}

DOI: 10.1103/PhysRevB.82.125319

PACS number(s): 78.67.-n, 78.20.Bh, 73.20.At, 78.55.Cr

\section{INTRODUCTION}

The realization of new optoelectronic devices exploiting the properties of InN, such as its direct band gap in the infrared range $e^{1,2}$ and high electron mobilities, ${ }^{3}$ relies on the ability to achieve both $n$-type and $p$-type doping. ${ }^{4}$ The latter has proven to be difficult as InN presents an exceptional propensity to unintentional $n$-type doping with native defects acting as donors. High free-electron densities increase the Fermi level and blueshift the absorption edge energy in $\mathrm{InN}$ films. ${ }^{5}$ This blueshift in the absorption spectra, the so-called Burstein-Moss shift, ${ }^{6}$ cannot be compensated by the bandgap renormalization resulting from electron-electron interaction. ${ }^{7}$ In addition to the bulk free-electron concentration, an electron accumulation layer forms at the (0001) polar surface of wurtzite InN. ${ }^{8-10}$ This is an intrinsic effect in InN caused by the pinning of the Fermi level above the conduction band minimum by donorlike surface states. For nonpolar surfaces, on the other hand, there is some controversy about the universality of the downwards band bending. While electrons accumulation is reported for both polar and non polar surfaces in samples exposed to air, ${ }^{9}$ flat electronic bands at the surfaces have been observed by scanning photoelectron spectroscopy performed on $a$-plane $\mathrm{InN}$ surfaces cleaved in high vacuum. ${ }^{11}$ However, it cannot be excluded that the quality of the cleavage has an influence on the electronic properties measured in Ref. 11. First-principles calculations confirmed the presence of positively charged surface states at the (0001) polar surface of wurtzite InN and concluded that stoichiometry could vary the character of nonpolar surface states. ${ }^{12}$

Recently, high-crystalline quality vertically self-oriented InN nanowires (NWs) have been obtained by plasmaassisted molecular-beam epitaxy (MBE). ${ }^{13-16}$ The increased surface-to-volume ratio of the columnar morphology opens up new functionalities for these nanostructures and favors their use in optical sensors and solar cells. At the same time, the inhomogeneous distribution of free electrons should be more evident. Indeed, the electrical and optical response of InN NWs show clear differences between the NWs inner cores and their surfaces. For instance, electrical measurements in single InN NWs have shown that transport takes place mainly at the lateral surface for NW diameters smaller than $\sim 60 \mathrm{~nm} .{ }^{17-19}$ On the other hand, a careful investigation of the photoluminescence (PL) peaks of InN NWs provided indirect evidences of a surface layer with degenerate electrons which is optically inactive. ${ }^{20}$ Finally, the coexistence of a plasmon-coupled mode and longitudinal optical modes in the Raman spectra ${ }^{20,21}$ indicated that while the NW core remains intrinsic, high electron densities are present at their lateral walls. These experimental results point out a complex interplay between the NW surface and core regions, where the free-electron densities should differ considerably.

In order to extract quantitative information about the spatial distribution of electron densities in InN NWs further efforts are required. Recently, Khanal et al. ${ }^{22}$ estimated the Fermi level relative to the conduction band at the surface of InN NWs from transconductance measurements and used an electrostatic model to calculate the charge distribution inside the NW. A much simpler approach involves measuring the absorption spectrum. This has been applied successfully in InN layers. ${ }^{23}$ For diffusive samples grown on opaque substrates, however, it is not possible to perform standard absorption measurements. In such cases, PL excitation (PLE) is accepted as a simple alternative, although the equivalence of PLE and absorption spectra is restrained to certain conditions. In general, both spectra are equivalent if photoexcited electron-hole pairs relax efficiently to the emitting state independently of their energy. ${ }^{24}$ An appropriate theoretical model is also necessary to extract the electron distribution from the absorption spectrum of InN NWs. While several studies have addressed the effect of a planar accumulation layer in the electronic structure of InN thin films, ${ }^{25-27}$ a model taking into account the columnar morphology is still lacking. 
TABLE I. Substrate temperature, In-BEP, $N_{2}^{\text {flux }}$ and $\mathrm{rf}$ power of the plasma source $\left(P_{\mathrm{rf}}\right)$ employed in the growth process of the series of NWs and the layer. The deposition rate $\left(_{d}\right)$ in units of InN monolayers per second and the growth time $\left(t_{g}\right)$, are also summarized.

\begin{tabular}{|c|c|c|c|c|c|}
\hline Sample & $\begin{array}{c}T_{\mathrm{s}} \\
\left({ }^{\circ} \mathrm{C}\right)\end{array}$ & $\begin{array}{c}\text { In-BEP } \\
\left(10^{-8} \text { mbar }\right)\end{array}$ & $\begin{array}{l}N_{2}^{\text {flux }} / P_{\text {rf }} \\
(\mathrm{sccm} / \mathrm{W})\end{array}$ & $\begin{array}{c}v_{d} \\
(\mathrm{ML} / \mathrm{s})\end{array}$ & $\begin{array}{c}t_{g} \\
(\mathrm{~min})\end{array}$ \\
\hline G532 & 500 & 1.5 & $1.5 / 450$ & 0.015 & 400 \\
\hline G041 & 500 & 1.5 & $1.5 / 400$ & 0.015 & 300 \\
\hline G044 & 500 & 3.0 & $1.5 / 450$ & 0.031 & 300 \\
\hline G136 & 475 & 3.0 & $1.5 / 450$ & 0.031 & 300 \\
\hline Layer & 450 & 1.5 & $1.5 / 400$ & 0.928 & 60 \\
\hline
\end{tabular}

In this work, the spatial distribution of degenerate electrons and its effects in the optical response of InN NWs are investigated. The optical absorption edge measured by PLE and the PL spectra of different sets of NWs are compared with those of an InN layer. The energy and lineshape of the PLE spectra are analyzed in comparison with the absorption spectrum resulting from a self-consistent calculation of the electronic structure.

The paper is organized as follows. Section II contains a brief description of the growth conditions, morphology, and crystalline quality of the investigated InN NWs. The results of the PL and PLE measurements are presented in Sec. III. A theoretical model for the degenerate electron distribution inside a cylindrical InN $\mathrm{NW}$ is developed in Sec. IV. The former model is used in the discussion of the experimental results in Sec. V. Finally, Sec. VI summarizes the main conclusions of this work.

\section{SAMPLES AND EXPERIMENTAL SETUP}

Four different samples containing InN NWs grown by plasma-assisted MBE on $\mathrm{Si}(111)$ substrates have been investigated. NWs self-oriented along the $c$ axis of the wurtzite structure were obtained keeping nitrogen-rich conditions during the growth process. The substrate temperature $\left(T_{\mathrm{s}}\right)$, the indium beam equivalent pressure (In-BEP) and the nitrogen flux $\left(N_{2}^{\text {flux }}\right)$ conditions were slightly varied obtaining different NW morphologies. The values of the parameters employed during the growth of each studied sample are summarized in Table I. InN NWs grown at the lowest $T_{\mathrm{s}}$ (G136) show a bimodal aspect ratio (height/diameter) distribution, with average diameters of $\sim 40 \mathrm{~nm}$ and $\sim 80 \mathrm{~nm}$, respectively. In contrast, an average diameter of $\sim 100 \mathrm{~nm}$ was measured for samples G041, G044, and G532. NW diameters presented a large dispersion, with standard deviations as large as $40 \mathrm{~nm}$. More details about the growth process and NW morphologies can be found elsewhere. ${ }^{16,20}$ A fifth sample with an InN layer $690 \pm 20 \mathrm{~nm}$ thick grown by plasma-assisted MBE on a bare sapphire substrate was also investigated. All samples were previously characterized by Raman scattering and showed very narrow $E_{2}^{h}$ phonon peaks, indicating a high-crystalline quality. ${ }^{20}$

PL and PLE measurements were carried out in a closecycle He cryostat kept at low temperature $(\sim 10 \mathrm{~K})$. A $50 \mathrm{~W}$ tungsten halogen lamp combined with a 1-m single grating monochromator served as tunable light source for both PL and PLE measurements. The emission from the NWs was dispersed by a $0.75-\mathrm{m}$ double monochromator and detected with a liquid-nitrogen cooled InAs photodiode. The spectral resolution of the PL and PLE experiments was around $1.5 \mathrm{meV}$.

\section{EXPERIMENTAL RESULTS}

In order to identify the optical signatures of the columnar morphology, the comparison between the PL and PLE spectra of a representative InN NW sample and of the InN epilayer is presented in Fig. 1. The PL spectrum of the InN NWs exhibits a single peak centered at higher energy than the InN layer one, with a large broadening as compared to those of GaN NWs ensemble $(\sim 1.5 \mathrm{meV}) .{ }^{28}$ The PLE spectra, obtained detecting at the PL maximum, show no excitonic peaks neither for the layer nor for the InN NWs. However, some noticeable differences can be observed between them. First of all, comparing their lineshapes, the PLE spectrum of the InN layer exhibits a sharp rise and its intensity remains almost constant above certain energy while the PLE intensity increases continuously and smoothly with the excitation energy for InN NWs. Regarding the absorption edge energy, obtained by linear extrapolation of the low-energy side of the PLE spectra, the InN NWs have a higher edge energy than the InN epilayer, and higher than the $0.67 \mathrm{eV}$ band-gap reported for InN at low temperatures. ${ }^{1,2,5}$ When compared with

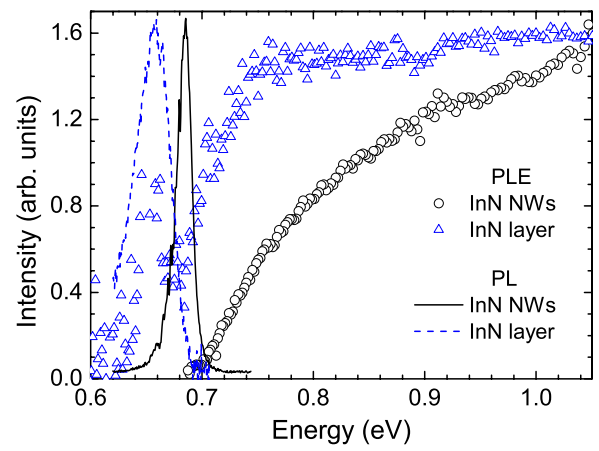

FIG. 1. (Color online) PL and PLE spectra of a representative NW sample (G041) and a high-crystalline quality InN layer measured at $10 \mathrm{~K}$. Open circles (triangles) and solid (dashed) line correspond to the PLE and PL spectra of NWs (layer), respectively. 

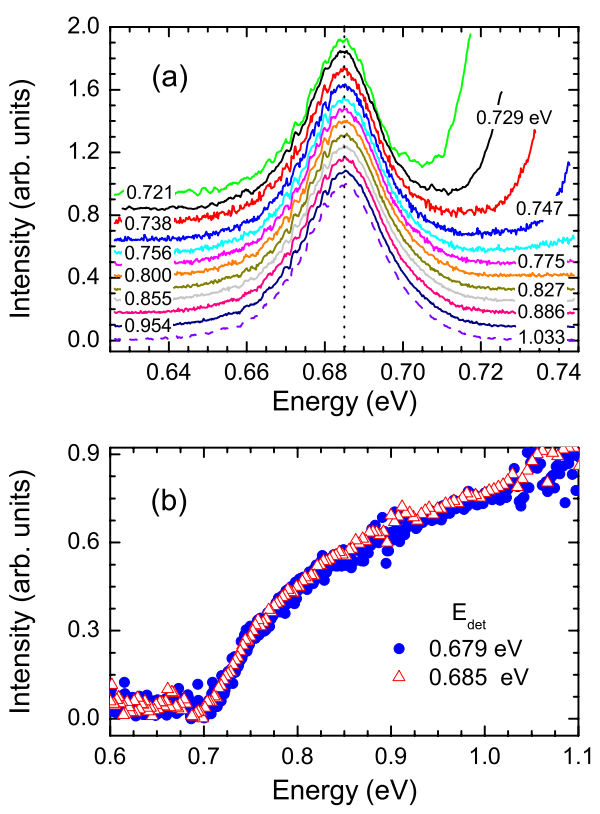

FIG. 2. (Color online) (a) Normalized PL spectra of sample G041 for different excitation energies indicated for each spectrum (values are given in electron volt). (b) PLE spectra of sample G041 detected at two different energies: $0.679 \mathrm{eV}$ (low energy tail) and $0.685 \mathrm{eV}$ (PL peak energy). One PLE spectrum was enhanced by a constant factor to make easier the comparison between them.

the PL spectra, a redshift (Stokes shift) between the PL peak and the PLE absorption edge is observed for the NWs while no noticeable Stokes shift is found for the InN layer.

The large broadenings of PL transitions of semiconductor nanostructures is often attributed to an inhomogeneous distribution of sizes of the nanoobjects. A more careful analysis of the InN NWs PL broadening has been carried out by varying the excitation energy over a wide range. By varying the excitation energy close to resonance, it is possible to excite selectively different sizes and reduce the emission linewidth in a process that has been referred to as spectral narrowing. ${ }^{29}$ Figure 2(a) shows no changes in the peak position and linewidth of the PL spectra of sample G041, obtained with excitation energies ranging from 0.72 to $1.03 \mathrm{eV}$. Similarly, Fig. 2(b) shows that neither the absorption edge nor the lineshape of the PLE spectra of sample G041 change as the detection energy is varied. According to these results, the broadening of the NWs PL peaks should correspond to the natural linewidth of the optical transitions, or in other words, to their homogeneous broadening.

Figure 3 gathers the normalized PL and PLE spectra of the series of InN NW samples. The fits of the PLE with the calculated absorption spectra are also included as solid lines and will be discussed in Sec. V. Some interesting differences can be observed among the experimental PLE spectra. First of all, the absorption edge energy varies over $45 \mathrm{meV}$ from sample to sample. A subtler difference can be found in the slope of the PLE onset at low energies which increases for increasing absorption edge energy. The differences described above cannot be attributed neither to confinement effects nor to changes in the average radius of the NWs. Notice that

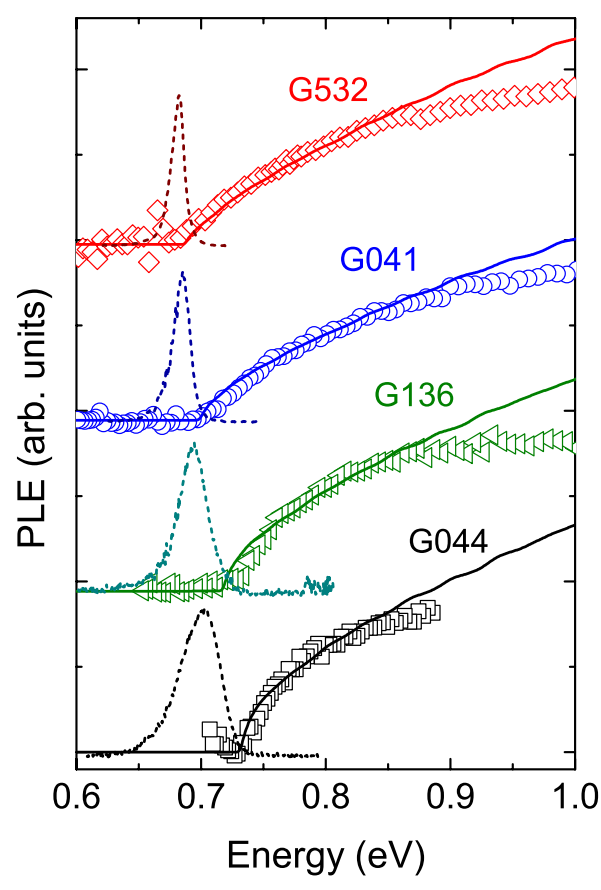

FIG. 3. (Color online) PL (dashed line) and PLE (open symbols) spectra detected at the PL peak energy for the different InN NW samples. Spectra are shifted along the vertical axis. Calculated absorption spectra (solid lines) showing the best agreement with the experimental ones are also included.

sample G136 contains the thinnest NWs of the series ( $\sim 40 \mathrm{~nm}$ thick). On the other hand, the energy and full width at half maximum (FWHM) of the PL peak also change with the absorption edge energy, as can be observed in Fig. 3.

Table II summarizes the absorption edge energy, the energy and FWHM of the PL peaks, and the Stokes shift for the InN NWs samples and the layer. The energy of the PL peak increases as the absorption edge energy increases but not with the same rate. Thus, the Stokes shift increases with the absorption edge energy of the sample. The FHWM of the PL peak also increases with the PLE absorption edge energy for the NWs. The InN layer breaks this behavior, showing a broader peak and a negligible Stokes shift. Again no correlation with the NW radius can be invoked. This behavior resembles, however, the previously reported correlation between the PL peak energy and linewidth of InN NWs, which

TABLE II. PLE absorption edge energy $\left(E_{0}^{\mathrm{PLE}}\right)$, energy $\left(E^{P L}\right)$ and FWHM of the PL peak, and Stokes shift for the series of InN NWs samples and the layer studied.

\begin{tabular}{lcccc}
\hline \hline Sample & $\begin{array}{c}E_{0}^{\mathrm{PLE}} \\
(\mathrm{meV})\end{array}$ & $\begin{array}{c}E^{\mathrm{PL}} \\
(\mathrm{meV})\end{array}$ & $\begin{array}{c}\text { FWHM } \\
(\mathrm{meV})\end{array}$ & $\begin{array}{c}\text { Stokes shift } \\
(\mathrm{meV})\end{array}$ \\
\hline G532 & $689 \pm 3$ & 682 & 12 & 7 \\
G041 & $699 \pm 3$ & 685 & 16 & 14 \\
G136 & $715 \pm 5$ & 694 & 25 & 21 \\
G044 & $728 \pm 4$ & 702 & 39 & 26 \\
Layer & $657 \pm 6$ & 657 & 39 & 0 \\
\hline \hline
\end{tabular}


has been attributed to changes in the electron accumulation layer at the nonpolar surfaces of the NWs. ${ }^{14,20}$

\section{THEORETICAL MODEL}

In order to establish the effect of free electrons on the optical absorption of InN NWs, a theoretical model that allows quantitative simulations of the charge and potential distribution within the NW has been developed. For the sake of simplicity, the NW is modeled as an infinite cylinder of radius $R$, and associated cylindrical coordinates $(r, \varphi, z)$ will be used throughout. Following the work of Klochikhin et al. ${ }^{25,26}$ and King et al. ${ }^{27}$ implemented for InN epilayers, we assume the presence of donorlike surface states. Surface states located above the Fermi level release their electrons to the InN conduction band, therefore providing a free-electrons population inside the wire, and a charge density $N_{s s}^{+}$at its surface $(r=R)$. The presence of a uniform ionized donor bulk doping (concentration $N_{D}^{+}$) is also assumed.

The charge and electrostatic potential profiles of the system can be obtained by following an iterative procedure for the self-consistent solution of the Schrödinger-Poisson system of equations ${ }^{30}$

Step 1. The NW confined electron-energy spectrum and wave functions are obtained within the effective-mass approximation by solving a Schrödinger-type equation with parabolic isotropic effective mass $m_{e}^{*}$. Despite the reported nonparabolicity of $\mathrm{InN}$ far from the $\Gamma$ point, ${ }^{31}$ the parabolic approximation has been shown to be valid in the range of energies where the model will be applied. ${ }^{32}$ Initially the potential energy $V(r)$ is constant and equal to the InN conduction band edge $\left(E_{c}\right)$, and hard-wall (infinite potential) boundary condition at $r=R$ is assumed throughout all the calculations.

Step 2. The calculated spectrum of single-electron quantum states is subsequently filled with the available electrons taking into account the Fermi-Dirac statistics. This allows to calculate the electron density as ${ }^{33}$

$$
n(r)=\sum_{\lambda} N_{\lambda}\left|\psi_{\lambda}(r)\right|^{2},
$$

where the index $\lambda$ contains the necessary quantum numbers to specify the one-dimensional quantum subbands. In the particular case of cylindrical symmetry confinement, $\lambda$ $=(n, l)$, and $n=(1,2, \ldots)$ the radial quantum number and $l$ $=(0, \pm 1, \ldots)$ is the $z$ component of the angular momentum.

The subband thermal occupancy is given by the factor

$$
N_{\lambda}=\sqrt{\frac{2 m_{c}^{*} k_{B} T}{\pi \hbar^{2}}} F_{-(1 / 2)}\left(\frac{E_{F}-E_{\lambda}}{k_{B} T}\right),
$$

where in Eqs. (1) and (2) $\psi_{\lambda}(r)$ and $E_{\lambda}$ are the envelope radial wave function and energy, of the $\lambda$ subband edge state $(k=0)$, respectively, and $F_{-(1 / 2)}(x)$ is the semi-integer Fermi integral. ${ }^{34}$ In contrast with other methods, such as the modified Thomas-Fermi approximation, ${ }^{27}$ the contribution of all the subbands to the electron density is explicitly calculated in the present approach. ${ }^{35}$

The Fermi level $E_{F}$ in Eq. (2), which sets the occupancy of the conduction band, is determined by taking into account that the total charge and must be equal to the charge provided by the donor impurities density and by the surface states density, i.e.,

$$
\int_{0}^{R} n(r) r d r=2 \pi R N_{s s}^{+}+\pi R^{2} N_{D}^{+}
$$

This nonlinear equation for $E_{F}$ is solved by the NewtonRaphson method.

Step 3. The charge density -en $(r)$ connects with the Poisson equation for the electrostatic potential

$$
\frac{1}{r} \frac{d}{d r}\left(r \frac{d \phi(r)}{d r}\right)=-\frac{e}{\varepsilon_{0} \varepsilon_{r}}\left[N_{D}^{+}-n(r)\right] .
$$

The presence of the ionized surface states with density $N_{s s}^{+}$ imposes the boundary condition for the potential

$$
\left.\frac{d \phi(r)}{d r}\right|_{r=R}=\frac{N_{s s}^{+}}{\varepsilon_{0} \varepsilon_{r}} .
$$

Once Eq. (4) is solved, one can construct a new potential energy as $V(r)=E_{c}-e \phi(r)$ and go back to Step 1 . The procedure is iterated with the method of successive over-relaxation until self-consistency is achieved, i.e., until the convergence in the charge-density profile is reached. ${ }^{36}$ The Schrödinger and Poisson differential equations are treated by the finite differences method adapted to cylindrical geometry, and avoiding the singularity at the origin. ${ }^{37}$ Once the selfconsistent problem has been solved, the final output are the conduction band edge profile, the electron distribution, and the Fermi level.

In order to study the absorption spectrum of the NWs, it is also necessary to calculate the valence-band electronic spectrum. To do so, a parabolic anisotropic band has been assumed (effective masses $m_{v, \|}^{*}=1.85, m_{v, \perp}^{*}=2.8$ ), ${ }^{38}$ whose edge varies locally due to the presence of the electrostatic potential induced by the electron distribution: $E_{v}-e \phi(r)$. Ionized acceptor states are not considered because their relative low density when compared with that of the valence band. The valence-band states are then calculated within the effective-mass approximation by solving once the corresponding Schrödinger-type equation.

Then, the optical absorption spectrum $\alpha(\hbar \omega)$ can be computed directly by means of the expression ${ }^{39}$

$$
\alpha(\hbar \omega) \propto \sum_{\lambda_{c}, \lambda_{v}} P_{\lambda_{c}, \lambda_{v}}^{2} g\left[\hbar \omega-\left(E_{\lambda_{c}}-E_{\lambda_{v}}\right)\right],
$$

where

$$
P_{\lambda_{c}, \lambda_{v}}=\int_{0}^{R} r d r \psi_{\lambda_{c}}(r) \psi_{\lambda_{v}}(r)
$$

is the oscillator strength for a transition from the valence subband $\lambda_{v}=\left(n_{v}, l\right)$ to the conduction subband $\lambda_{c}=\left(n_{c}, l\right)$ (note the angular momentum conservation); the joint density of states is 


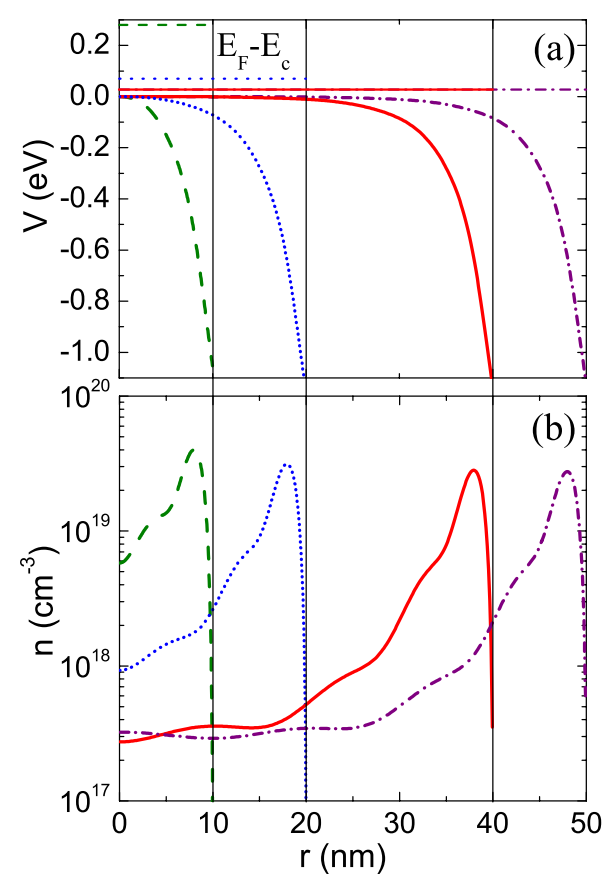

FIG. 4. (Color online) (a) Conduction band edge, $V(r)=E_{c}$ $-e \phi(r)$ and (b) electron density profiles for InN NWs with $N_{D}^{+}$ $=3.0 \times 10^{17} \mathrm{~cm}^{-3}$ and $N_{s s}^{+}=1.0 \times 10^{13} \mathrm{~cm}^{-2}$, and different radius ranging from 10 (dashed line) to $50 \mathrm{~nm}$ (dashed-dotted line). The Fermi level of each NW is also indicated. The energy scale is referred to $E_{c}$.

$$
g(\epsilon)=\sqrt{\frac{\mu}{\pi \hbar^{2} \epsilon}}\left\{1+\exp \left[\frac{\frac{\mu}{m_{c}^{*}} \epsilon-\left(E_{F}-E_{\lambda_{c}}\right)}{k_{B} T}\right]\right\}^{-1},
$$

which takes into account the filling of the conduction band, and the electron-hole reduced mass $\mu=\left(m_{c}^{*} m_{v, \perp}^{*}\right) /\left(m_{c}^{*}\right.$ $\left.+m_{v, \perp}^{*}\right) .{ }^{39,40}$ For the calculations, an effective electron mass of $m_{c}^{*}=0.05 m_{0},{ }^{41}\left(m_{0}\right.$ is the free-electron mass) and a fundamental band-gap of $E_{g}=0.67 \mathrm{eV}$ reported for bulk $\mathrm{InN}$ were used, considering the large NW sizes. It should be mentioned that these values are in constant revision, and their status have a provisional character. The temperature was fixed at 10 $\mathrm{K}$ to match that of the optical measurements presented in this work.

\section{A. Effect of the nanowire radius}

The suitability of our approach has been illustrated for InN NWs of different radii. A constant $N_{D}^{+}=3.0$ $\times 10^{17} \mathrm{~cm}^{-3}$ was assumed, as reported for high-quality $\mathrm{InN}$ layers. ${ }^{42} N_{s s}^{+}$was set at $1.0 \times 10^{13} \mathrm{~cm}^{-2}$, in the range of the typical values reported for InN polar surfaces. ${ }^{10,43,44}$ Figures 4(a) and 4(b) show the profiles of the conduction band edge, $V(r)=E_{c}-e \phi(r)$, and the electron concentration, $n(r)$, corresponding to InN NWs with $R=10,20,40$, and $50 \mathrm{~nm}$. These radii are within the values reported for the MBE-grown $\mathrm{InN}$ NWs. ${ }^{16}$ The Fermi level is also indicated for each case. For all the NWs, the conduction band profile falls from 0 at the inner part of the NW to around $-1 \mathrm{eV}$ at the surface. This

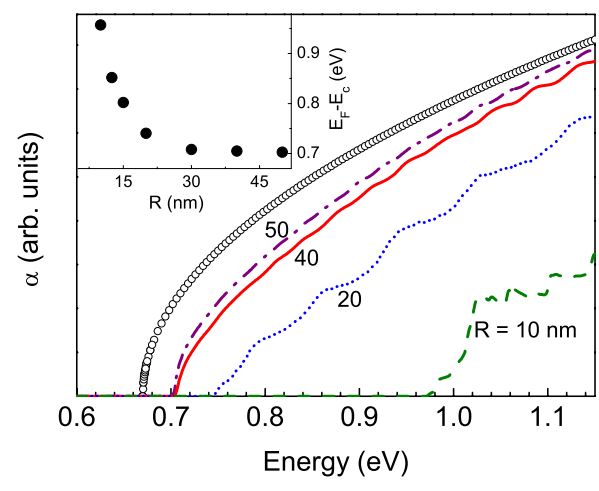

FIG. 5. (Color online) Optical absorption spectra for the same InN NWs considered in Fig. 4. $R=10$ (dashed line), 20 (dotted line), 40 (solid line), and $50 \mathrm{~nm}$ (dashed-dotted line). The $E^{1 / 2}$ ideal dependence of the absorption of intrinsic bulk InN with a band-gap energy of $0.67 \mathrm{eV}$ is also displayed (open circles). The inset shows the evolution of the absorption edge with the radius.

transition occurs abruptly in the near-surface region $(R-r$ $\$ 10 \mathrm{~nm}$ ) causing an accumulation of free electrons at the surface region. However, while for wider NWs $(R$ $>30 \mathrm{~nm}$ ) the free electrons are remarkably concentrated at the surface and a relatively low concentration is found in the NW inner core, the reduction in the radius implies a more uniform distribution of free electrons. Furthermore, for lower $R$, the conduction band edge loses the planar shape in the NW center, adopting a parabolic profile. The Fermi level relative to the conduction band edge at the center of the NW, $E_{F}^{0}=E_{F}-V(r=0)$, reaches a value of $\sim 0.29 \mathrm{eV}$ for $R$ $=10 \mathrm{~nm}$, and decreases for increasing $R$ down to an almost constant value of $\sim 0.03 \mathrm{eV}$ for $R>30 \mathrm{~nm}$. As an effect of the low temperature $(10 \mathrm{~K})$, Friedel-type oscillations are present for all the $n(r)$ distributions. ${ }^{35}$

The calculated optical absorption of InN NWs with different radii is presented in Fig. 5. The absorption for intrinsic bulk InN within the parabolic approximation is also shown (open circles). The most relevant effects of decreasing $R$ are, first, a noticeable blueshift of the absorption edge (see the inset of Fig. 5). Since quantum confinement is negligible for all the considered NW sizes, this displacement is produced mainly by the conduction band occupancy. In the second place, the shape and intensity of the absorption of thinner NWs deviate from the $E^{1 / 2}$ bulk absorption.

Although the role of the NW radius can be determined for its optical properties, other intrinsic properties of the NWs could also affect their optical response. For instance, differences in the concentration of bulk donor impurities and surface charge are expected to change the optical properties of these nanostructures.

\section{B. Effect of donor impurities and surface charge}

The conduction band profile and electron concentration in an InN NW with $R=40 \mathrm{~nm}$ have been calculated for different values of $N_{D}^{+}$and $N_{s s}^{+}$. Figures 6(a) and 6(b) show that for a fixed $N_{D}^{+}$value, higher values of $N_{s s}^{+}$result in a more pronounced bending of the conduction band and a larger difference between the electron densities at the surface and in the 


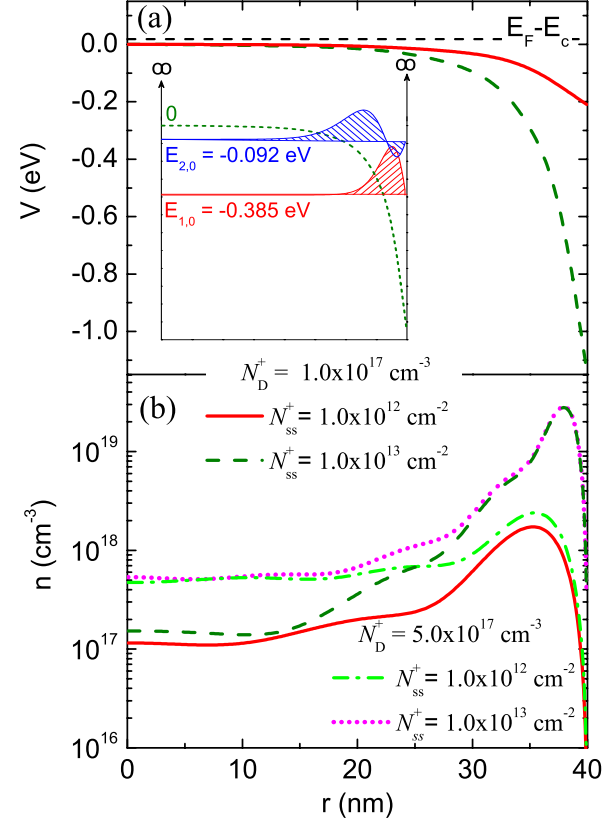

FIG. 6. (Color online) (a) Conduction band and (b) electron concentration profiles of a $40 \mathrm{~nm}$ radius $\mathrm{InN} \mathrm{NW}$ with $N_{D}^{+}=1.0$ $\times 10^{17} \mathrm{~cm}^{-3}$ and two different values of $N_{s s}^{+}: 1.0 \times 10^{12}$ and 1.0 $\times 10^{13} \mathrm{~cm}^{-2}$. The electron density profiles are also plotted for $N_{D}^{+}$ $=5.0 \times 10^{17} \mathrm{~cm}^{-3}$. The inset shows the conduction band profile for the higher $N_{s s}^{+}$value and the first two conduction band states $(l=0)$.

core of the NW. On the other hand, fixing the $N_{s s}^{+}$value, the bending of the conduction band is not affected by the decrease in $N_{D}^{+}$(not shown in the figure) while the differences between the electron concentration at the surface and in the inner part of the NW increase. The wave functions of the first two conduction band states with $l=0$ are depicted in the inset of Fig. 6.

Figure 7 shows the optical absorption calculated for the same set of NWs considered in Fig. 6. The $E^{1 / 2}$ ideal dependence of the absorption for intrinsic bulk $\mathrm{InN}$ is also plotted for comparison. This figure shows that distinctive optical features can be associated to variations in the bulk impurity concentration and surface charge density of the NWs. First of

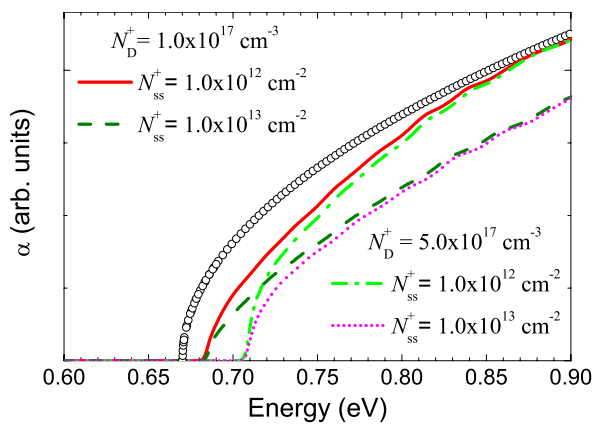

FIG. 7. (Color online) Calculated absorption spectra of a $40 \mathrm{~nm}$ radius $\mathrm{InN} \mathrm{NW}$ for two different values of $N_{D}^{+}: 1.0 \times 10^{17}$ and 5.0 $\times 10^{17} \mathrm{~cm}^{-3}$; and two different values of $N_{s s}^{+}: 1.0 \times 10^{12}$ and 1.0 $\times 10^{13} \mathrm{~cm}^{-2}$. The $E^{1 / 2}$ ideal dependence of the absorption for intrinsic bulk $\mathrm{InN}$ (open circles) is also displayed.

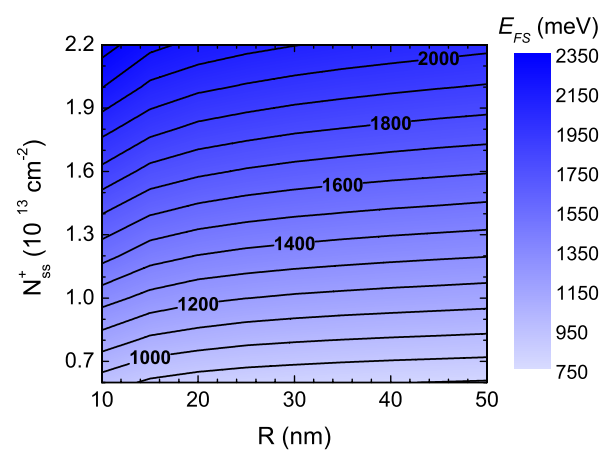

FIG. 8. (Color online) Mapping of $E_{F S}$ as a function of both $N_{s s}$ and radius of the NW.

all, increasing $N_{D}^{+}$blueshifts the absorption edge due to the Burstein-Moss effect. On the other hand, $N_{s s}^{+}$values do not contribute noticeable to the absorption edge shift but modify significantly the absorption onset and its absolute intensity. A slower rise is obtained for higher $N_{s s}^{+}$due to the larger spatial separation of electrons and holes caused by the larger potential bending at the surface reported in Fig. 6(a).

The value of the Fermi level measured relative to the conduction band edge at the surface of the NW, $E_{F S}=E_{F}$ $-V(r=R)$, has been recently determined for InN single NWs with different $R$ from electrolyte-gating measurements. ${ }^{22}$ Considering the experimental accessibility to this magnitude, $E_{F S}$ as a function of $N_{s S}^{+}$and $R$ has been calculated using our theoretical model. Figure 8 shows a map of $E_{F S}$ as a function of both $N_{s s}^{+}$and $R$. These results can be used to determine $N_{s s}^{+}$ of a single NW from its reported value of $E_{F S}$ for any radii.

\section{DISCUSSION}

Before dealing with the experimental results of the $\mathrm{InN}$ NWs, the optical properties of the InN layer should be briefly discussed. First of all, the PL of the InN layer presents a 39 meV FWHM and does not follow the trend with the PL energy found for the InN NWs (see Table II). Large linewidth values are often an indication of inhomogeneities in the sample. Further evidences of the inhomogeneous nature of the InN layer emission are found in the PLE spectra recorded at different detection energies (not shown here). On the contrary to our observations for the InN NWs, the absorption edge moves in energy for different detection energies. In the case of $\mathrm{InN}$, free electron concentration increases the emission linewidth following a power law, as shown by a recent study. ${ }^{45}$ According to this work, an inhomogeneous broadening of $\sim 10 \mathrm{meV}$ and a moderate electron density (between $1.0 \times 10^{17}$ and $5.0 \times 10^{17} \mathrm{~cm}^{-3}$ ) would account for the measured FWHM of the current epilayer.

The PLE spectrum of the InN layer of Fig. 1 exhibits a sharper absorption edge with a decrease in the intensity above certain threshold energy, similarly to previous reported results. ${ }^{46,47}$ Such lineshape resembles that of the excitonic continuum onset. Although Coulomb interaction between a localized hole in the valence band and an electron in the 
TABLE III. Absorption edge energy $\left(E_{0}\right), E_{F S}, N_{D}^{+}$, and $N_{s s}^{+}$that provided the best agreement between experimental and theoretical spectra of the samples studied in this work.

\begin{tabular}{lcccc}
\hline \hline Sample & $\begin{array}{c}E_{0} \\
(\mathrm{eV})\end{array}$ & $\begin{array}{c}E_{F S} \\
(\mathrm{eV})\end{array}$ & $\begin{array}{c}N_{D}^{+} \\
\left(\mathrm{cm}^{-3}\right)\end{array}$ & $\begin{array}{c}N_{s S}^{+} \\
\left(\mathrm{cm}^{-2}\right)\end{array}$ \\
\hline G532 & 0.687 & 2.36 & $0.8 \times 10^{17}$ & $2.5 \times 10^{13}$ \\
G041 & 0.696 & 2.20 & $2.0 \times 10^{17}$ & $2.4 \times 10^{13}$ \\
G136 & 0.711 & 1.66 & $5.0 \times 10^{17}$ & $1.7 \times 10^{13}$ \\
G044 & 0.726 & 1.49 & $7.5 \times 10^{17}$ & $1.4 \times 10^{13}$ \\
Layer & 0.657 & & & \\
\hline \hline
\end{tabular}

Fermi sea is not completely screened for free-carrier concentrations below $\sim 1.0 \times 10^{18} \mathrm{~cm}^{-3}$ for $\mathrm{InN},{ }^{48}$ no excitonic resonance is observed in the PLE spectra of Fig. 1. Once excitonic effects have been disregarded, another possible explanation has been pointed out which invokes the creation of mobile holes above a certain photon-energy threshold. When both the electron and hole are mobile, the probability of separation of an electron-hole pair and, hence, the probability of nonradiative recombination increases. As a result, the PLE efficiency substantially decreases above the threshold energy. ${ }^{47}$

The former argument brings the debate about the validity of considering that PLE and absorption spectra are equivalents. At low-excitation energies, the PLE onset follows the absorption one because nonradiative recombination is negligible. However, at higher excitation energies the activation of nonradiative traps reduces the efficiency of the relaxation to the ground state. ${ }^{24}$ Therefore, PLE and absorption spectra are comparable in the low energy range only.

Several distinctive features appearing in the PL and PLE spectra of InN NWs described in Sec. III indicate a stronger effect of free electrons in comparison to bulk samples. Furthermore, differences in the absorption edge energy and shape, and in the PL energy and linewidth could be observed in the samples of the studied series (see Fig. 3 and Table II). The theoretical absorption spectra presented in Sec. IV also showed a clear dependence in some relevant characteristics of the NWs, i.e., radius, doping level, and surface charge. The NW radius modifies the distribution of free electrons and therefore the absorption edge energy, as reported in Figs. 4 and 5. However, most of the NWs investigated here have large radii $(R>30 \mathrm{~nm})$ and the evolution of the optical parameters presented in Table II did not evidence any correlation between absorption edge and $R$ for $R>30 \mathrm{~nm}$. Therefore, $N_{D}^{+}$and $N_{s s}^{+}$are expected to dominate the absorption spectra of these NWs, and the differences found among the absorption spectra of the studied samples must be also a consequence of different values of these two parameters.

Fittings of the experimental PLE spectra with the calculated absorption spectra have been included as solid lines in Fig. 3. A fixed value of $R=40 \mathrm{~nm}$ has been assumed for all $\mathrm{NW}$ samples. The values of $N_{D}^{+}$and $N_{s s}^{+}$that provided the best agreement between PLE and theoretical spectra are reported in Table III. It should be pointed out that $N_{D}^{+}$and $N_{s s}^{+}$can be estimated independently, as they modify the absorption edge energy and its slope, respectively. The theoretical fittings show a good agreement with the experimental data at energies close to the PLE onset. At higher energies, the PLE intensity decreases presumably due to the activation of nonradiative recombination channels and deviates from the theoretical spectrum.

The values found for $N_{D}^{+}$are in good agreement with those cited in the literature and point out that InN NWs grown by plasma-assisted MBE contain a relatively low density of donor impurities. On the other hand, those obtained for $N_{s s}^{+}$vary from one sample to another suggesting that this parameter could be affected by the growth conditions. Such a result is expected from the theoretical predictions of Van der Walle and co-workers for nonpolar InN surfaces ${ }^{12}$ and imply that specific growth conditions can reduce the surface electron accumulation in these NWs. Finally, and according to the theoretical results of Fig. $6, N_{D}^{+}$and $N_{s s}^{+}$values in Table III support the idea of two distinctive regions in the NWs, and agree with the coexistence of plasma coupled and uncoupled LO modes in the Raman spectra of these nanostructures. ${ }^{20,21}$

Other aspects of the optical response also reinforce the proposed picture. For instance, the existence of a Stokes shift which increased in magnitude with the absorption edge energy can be understood from the fact that different processes lead to emission and absorption. While absorption involves a transition from an occupied state in the valence band into an empty state in the conduction band, emission occurs after a recombination between any free electron in the conduction band and a free or bound hole in the valence band. ${ }^{20,25,49}$ Both processes should follow wave-vector conservation. However, the momentum conservation selection rule in the recombination is broken by the localization of holes, which allows for the emission involving all the occupied states in the conduction band of degenerate InN samples. ${ }^{3}$ The lower probability for optical transitions at higher values of the wave vector explains why the PL peak appears at an energy between the conduction band minimum and the absorption edge, and why the Stokes shift increases for increasing absorption edge. The breakdown of the wave-vector conservation also accounts for the large homogeneous broadening (10-40 meV) and Burstein-Moss shift (15-40 meV) observed for all the NWs samples studied.

\section{CONCLUSIONS}

InN NWs grown under different conditions have been characterized by PL and PLE. Differences in the absorption edge and lineshape among the PLE spectra were observed. The absorption edge energy showed a correlation with both the energy and the FWHM of the PL peak. Finally, the large broadening of the PL peaks was found to be homogeneous. A theoretical model has been developed to calculate the distribution of degenerate electrons inside a NW. The columnar geometry introduced effects in both the electron density and in the self-consistent conduction band profile, with no equivalence in planar layers. NWs with $R>30 \mathrm{~nm}$ have shown a relatively low electron concentration at the inner core while a high density of degenerate electrons were accumulated at their lateral walls. The PLE spectra of the NWs 
ensemble have been reproduced by the calculated absorption spectra, leading to an estimated set of values of $N_{D}^{+}$and $N_{s s}^{+}$ for each sample. The estimated values of $N_{s s}^{+}$suggest that specific growth conditions can reduce the surface electron accumulation in these NWs. Large differences observed between the optical properties of a layer and NWs of InN can be understood by the strong effect of surface-to-volume ratio on the optical properties of $\mathrm{InN}$.

\section{ACKNOWLEDGMENTS}

This work was supported by the ERANET project "NanoSci-ERA: NanoScience in the European Research Area" and ACDET II of the EU FP6, and by the Ministry of Science and Innovation of Spain (Grant No. MAT200910350). J.S.-R. thanks the Ministry of Science and Innovation of Spain for the financial support through a FPU grant. F.I. thanks CAPES, FAPESP, and CNPQ.
${ }^{1}$ V. Yu. Davydov, A. A. Klochikhin, R. P. Seisyan, V. V. Emtsev, S. V. Ivanov, F. Bechstedt, J. Furthmüller, H. Harima, A. V. Mudryi, J. Aderhold, O. Semchinova, and J. Graul, Phys. Status Solidi B 229, R1 (2002).

${ }^{2}$ J. Wu, W. Walukiewicz, K. M. Yu, J. W. Ager III, E. E. Haller, H. Lu, W. J. Schaff, Y. Saito, and Y. Nanishi, Appl. Phys. Lett. 80, 3967 (2002).

${ }^{3}$ W. Walukiewicz, J. W. Ager III, K. M. Yu, Z. Liliental-Weber, J. Wu, S. X. Li, R. E. Jones, and J. D. Denlinger, J. Phys. D 39, R83 (2006).

${ }^{4}$ R. E. Jones, K. M. Yu, S. X. Li, W. Walukiewicz, J. W. Ager, E. E. Haller, H. Lu, and W. J. Schaff, Phys. Rev. Lett. 96, 125505 (2006).

${ }^{5}$ J. S. Thakur, Y. V. Danylyuk, D. Haddad, V. M. Naik, R. Naik, and G. W. Auner, Phys. Rev. B 76, 035309 (2007).

${ }^{6}$ E. Burstein, Phys. Rev. 93, 632 (1954).

${ }^{7}$ K. F. Berggren and B. E. Sernelius, Phys. Rev. B 24, 1971 (1981).

${ }^{8}$ I. Mahboob, T. D. Veal, C. F. McConville, H. Lu, and W. J. Schaff, Phys. Rev. Lett. 92, 036804 (2004)

${ }^{9}$ P. D. C. King, T. D. Veal, C. F. McConville, F. Fuchs, J. Furthmüller, F. Bechstedt, P. Schley, R. Goldhahn, J. Schörmann, D. J. As, K. Lischka, D. Muto, H. Naoi, Y. Nanishi, H. Lu, and W. J. Schaff, Appl. Phys. Lett. 91, 092101 (2007).

${ }^{10}$ P. D. C. King, T. D. Veal, C. S. Gallinat, G. Koblmüller, L. R. Bailey, J. S. Speck, and C. F. McConville, J. Appl. Phys. 104, 103703 (2008).

${ }^{11}$ C. L. Wu, H. M. Lee, C. T. Kuo, C. H. Chen, and S. Gwo, Phys. Rev. Lett. 101, 106803 (2008).

${ }^{12}$ C. G. Van de Walle and D. Segev, J. Appl. Phys. 101, 081704 (2007).

${ }^{13}$ J. M. Sánchez-García, J. Grandal, E. Calleja, S. Lazić, J. M. Calleja, and A. Trampert, Phys. Status Solidi B 243, 1490 (2006).

${ }^{14}$ T. Stoica, R. Meijers, R. Calarco, T. Richter, and H. Lüth, J. Cryst. Growth 290, 241 (2006); T. Stoica, R. J. Meijers, R. Calarco, T. Richter, E. Sutter, and H. Lüth, Nano Lett. 6, 1541 (2006).

${ }^{15}$ C.-H. Shen, H.-Y. Chen, H.-W. Lin, S. Gwo, A. A. Klochikhin, and V. Yu. Davydov, Appl. Phys. Lett. 88, 253104 (2006).

${ }^{16}$ J. Segura-Ruiz, N. Garro, A. Cantarero, C. Denker, F. Werner, J. Malindretos, and A. Rizzi, Phys. Status Solidi C 5, 1678 (2008); C. Denker, J. Malindretos, F. Werner, F. Limbach, H. Schuhmann, T. Niermann, M. Seibt, and A. Rizzi, ibid. 5, 1706 (2008).

${ }^{17}$ E. Calleja, J. Grandal, M. A. Sánchez-García, M. Niebelschütz, V. Cimalla, and O. Ambacher, Appl. Phys. Lett. 90, 262110
(2007).

${ }^{18}$ T. Richter, C. Blömers, H. Lüth, R. Calarco, M. Indlekofer, M. Marso, and T. Schäpers, Nano Lett. 8, 2834 (2008).

${ }^{19}$ F. Werner, F. Limbach, M. Carsten, C. Denker, J. Malindretos, and A. Rizzi, Nano Lett. 9, 1567 (2009).

${ }^{20}$ J. Segura-Ruiz, N. Garro, A. Cantarero, C. Denker, J. Malindretos, and A. Rizzi, Phys. Rev. B 79, 115305 (2009).

${ }^{21}$ S. Lazić, E. Gallardo, J. M. Calleja, F. Agulló-Rueda, J. Grandal, M. A. Sánchez-García, E. Calleja, E. Luna, and A. Trampert, Phys. Rev. B 76, 205319 (2007).

${ }^{22}$ D. R. Khanal W. Walukiewicz, J. Grandal, E. Calleja, and J. Wu, Appl. Phys. Lett. 95, 173114 (2009).

${ }^{23}$ J. Wu, W. Walukiewicz, S. X. Li, R. Armitage, J. C. Ho, E. R. Weber, E. E. Haller, H. Lu, W. J. Schaff, A. Barcz, and R. Jakiela, Appl. Phys. Lett. 84, 2805 (2004).

${ }^{24}$ P. Y. Yu and M. Cardona, Fundamental of Semiconductors (Springer-Verlag, Berlin, 1999).

${ }^{25}$ A. A. Klochikhin, V. Y. Davydov, I. Y. Strashkova, P. N. Brunkov, A. A. Gutkin, M. E. Rudinsky, H. Y. Chen, and S. Gwo, Phys. Status Solidi (RRL) 1, 159 (2007).

${ }^{26}$ A. A. Klochikhin, V. Y. Davydov, I. Y. Strashkova, and S. Gwo, Phys. Rev. B 76, 235325 (2007).

${ }^{27}$ P. D. C. King, T. D. Veal, and C. F. McConville, Phys. Rev. B 77, 125305 (2008).

${ }^{28}$ F. Furtmayr, M. Vielemeyer, M. Stutzmann, A. Laufer, B. K. Meyer, and M. Eickhoff, J. Appl. Phys. 104, 074309 (2008).

${ }^{29}$ O. I. Mićić, H. M. Cheong, H. Fu, A. Zunger, J. R. Sprague, A. Mascarenhas, and A. J. Nozik, J. Phys. Chem. B 101, 4904 (1997).

${ }^{30}$ M. Tadić and Z. Ikonić, Phys. Rev. B 50, 7680 (1994).

${ }^{31}$ W. Walukiewicz, S. X. Li, J. Wu, K. M. Yu, J. W. Ager III, E. E. Haller, H. Lu, and W. Schaff, J. Cryst. Growth 269, 119 (2004).

${ }^{32}$ S. Abe, T. Inaoka, and M. Hasegawa, Phys. Rev. B 66, 205309 (2002).

${ }^{33}$ A. Trellakis, A. T. Galick, A. Pacelli, and U. Ravaioli, J. Appl. Phys. 81, 7880 (1997).

${ }^{34}$ M. Goano, ACM Trans. Math. Softw. 21, 221 (1995).

${ }^{35}$ J. H. Luscombe, A. M. Bouchard, and M. Luban, Phys. Rev. B 46, 10262 (1992).

${ }^{36}$ W. H. Press, B. P. Flannery, S. A. Teukolsky, and W. T. Vetterling, Numerical Recipes in Fortran 77, 2nd ed. (Cambridge University Press, Cambridge, England, 1992).

${ }^{37}$ K. Mohseni and T. Colonius, J. Comput. Phys. 157, 787 (2000).

${ }^{38}$ A. Molina-Sánchez, A. García-Cristóbal, A. Cantarero, A. Terentjevs, and G. Cicero (unpublished).

${ }^{39}$ G. Grosso and G. Pastori Parravicini, Solid State Physics (Academic, London, 2000). 
${ }^{40}$ In the practical numerical results presented here, the function $1 / \sqrt{\epsilon}$ is substituted by $\mathfrak{R}(1 / \sqrt{\epsilon+i \gamma})$, with $\gamma=10 \mathrm{meV}$, to smooth the singularity around $\epsilon=0$.

${ }^{41}$ S. P. Fu and Y. F. Chen, Appl. Phys. Lett. 85, 1523 (2004).

${ }^{42}$ A. A. Klochikhin, V. Y. Davydov, V. V. Emtsev, A. V. Sakharov, V. A. Kapitonov, B. A. Andreev, H. Lu, and W. J. Schaff, Phys. Rev. B 71, 195207 (2005).

${ }^{43}$ L. Colakerol, T. D. Veal, H.-K. Jeong, L. Plucinski, A. DeMasi, T. Learmonth, P. A. Glans, S. Wang, Y. Zhang, L. F. J. Piper, P. H. Jefferson, A. Fedorov, T.-C. Chen, T. D. Moustakas, C. F. McConville, and K. E. Smith, Phys. Rev. Lett. 97, 237601 (2006).

${ }^{44}$ A. A. Gutkin, M. É. Rudinsky, P. N. Brunkov, A. A. Klochikhin, V. Y. Davydov, H. Y. Chen, and S. Gwo, Semiconductors 42, 1416 (2008)
${ }^{45}$ M. Moret, F. Ruffenach, O. Briot, and B. Gil, Appl. Phys. Lett. 95, 031910 (2009).

${ }^{46}$ V. Yu. Davydov, A. A. Klochikhin, V. V. Emtsev, D. A. Kurdyukov, S. V. Ivanov, V. A. Vekshin, F. Bechstedt, J. Furthmüller, J. Aderhold, J. Graul, A. V. Mudryi, H. Harima, A. Hashimoto, A. Yamamoto, and E. E. Haller, Phys. Status Solidi B 234, 787 (2002).

${ }^{47}$ V. Yu. Davydov and A. A. Klochikhin, Semiconductors 38, 861 (2004).

${ }^{48}$ M. Feneberg, J. Däubler, K. Thonke, R. Sauer, P. Schley, and R. Goldhahn, Phys. Rev. B 77, 245207 (2008).

${ }^{49}$ B. Arnaudov, T. Paskova, P. P. Paskov, B. Magnusson, E. Valcheva, B. Monemar, H. Lu, W. J. Schaff, H. Amano, and I. Akasaki, Phys. Rev. B 69, 115216 (2004). 